\title{
The Dispersion Rule of Fragments about the Asymmetric Shell
}

\author{
Liangliang Ding, Zhenduo Li, Minzu Liang, Xiangyu Li, and Fangyun Lu \\ College of Science, National University of Defense Technology, Changsha, Hunan 410073, China \\ Correspondence should be addressed to Xiangyu Li; xiangyulee@nudt.edu.cn
}

Received 8 September 2016; Accepted 29 December 2016; Published 6 February 2017

Academic Editor: Francesco Franco

Copyright (C) 2017 Liangliang Ding et al. This is an open access article distributed under the Creative Commons Attribution License, which permits unrestricted use, distribution, and reproduction in any medium, provided the original work is properly cited.

\begin{abstract}
In order to obtain the dispersion rule of fragments about the asymmetric shell subjected to internal blast loading, two different cross section structures, concave-shaped and convex-shaped, were carried out by experimental and numerical methods. The simulation results well coincided with the experimental results, and the spatial distribution and fragment velocity were obtained. The optimal curvatures for the different concave structures changed from $4 r$ to $6 r$ ( $r$ represents the charge radius), as the central angle of concave structure changed from $90^{\circ}$ to $120^{\circ}$. However, the optimal curvature changed weakly when the central angle of concave structure was larger than $120^{\circ}$. In addition, a formula which can rapidly predict the projection angle range was fitted for the convex structure. The conclusions can provide a reference for concave-shaped and convex-shaped structures to achieve a higher effectiveness of fragments.
\end{abstract}

\section{Introduction}

Cylindrical shell is widely used in the engineering field, especially in the aerospace and defense industry. A lot of researches about the damage of cylindrical shell with internal explosion have been undertaken. With the increase of charge mass, the cylindrical shell was torn into lots of fragments under the action of shock wave and detonation products. Gurney researched the dispersion rule of fragments about the cylindrical shell and presented the famous Gurney formula which can be used to evaluate the fragment velocity [1]. Taylor presented a semiempirical formula (Taylor formula) for the long cylindrical shell, and the projection angle of fragments can be obtained by using the Taylor formula [2]. Mott studied the spatial distribution and size of fragments about the tubular structure, which was based on the probability theory and perfectly plastic material model [3]. In order to broaden the application of Taylor formula, significant researches were carried out by various authors, and the researches mainly focused on the end effects and the projection angle of cylindrical shell [4-7]. A lot of researches on the fragment's dispersion characteristics of cylindrical shell have been carried out [8-12]. Gold [13] proposed a new design method for the asymmetric cylindrical structure, which can better improve the controllability and the damage power of fragments. Gong et al. [14] and Wang et al. [15] reported an empirical formula and a new method to estimate the projection angles and fragment velocities of D-shaped cylindrical shell. Although there were some researches on the fragment's dispersion characteristics of asymmetric shell, little attentions were devoted to the influence of cross section on the fragment's dispersion characteristics. Here, the asymmetric structure was defined as three types, namely, concaveshaped, convex-shaped, and D-shaped. This article mainly takes the concave-shaped and convex-shaped structure as the research objects to analyze the dispersion rule of fragments. The final conclusion can provide a reference for the concaveshaped and convex-shaped structure to achieve a higher effectiveness of fragments.

\section{Experiments}

2.1. Specimen. The concave-shaped and convex-shaped specimens used in the experiments are shown in Figure 1. The specimen contains four parts: upper endplate, lower endplate, TNT charge, and asymmetric shell; the material properties of them are listed in Table 1. The four parts have similar profile which consists of two pieces of circular arc; the central angle of one piece of circular arc is $240^{\circ}$ and the radius is $r$, while 


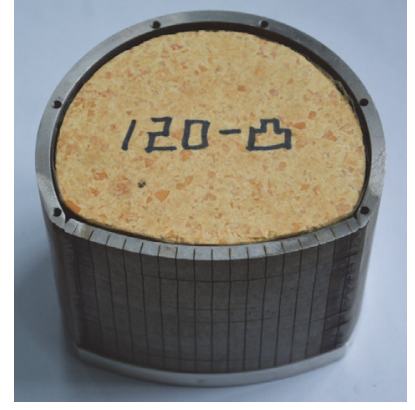

(a) Convex $-120^{\circ}$

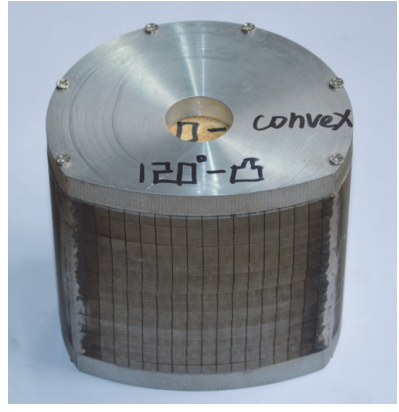

FIGURE 1: Specimens of concave- $120^{\circ}$ and convex- $120^{\circ}$ structures.

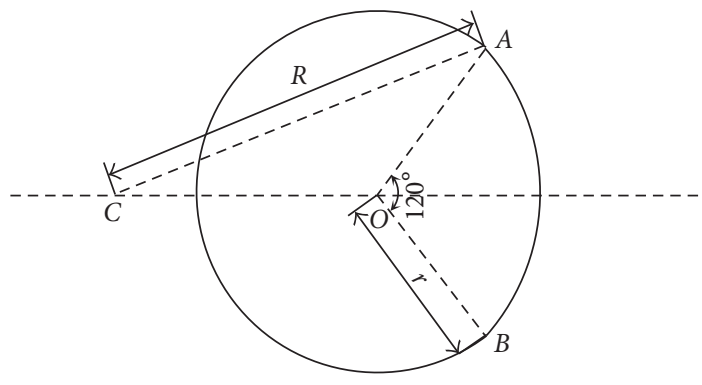

(a) Convex $-120^{\circ}$
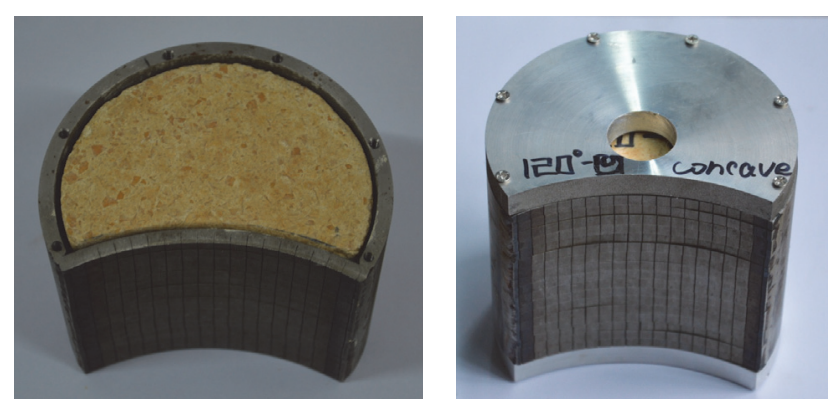

(b) Concave- $120^{\circ}$

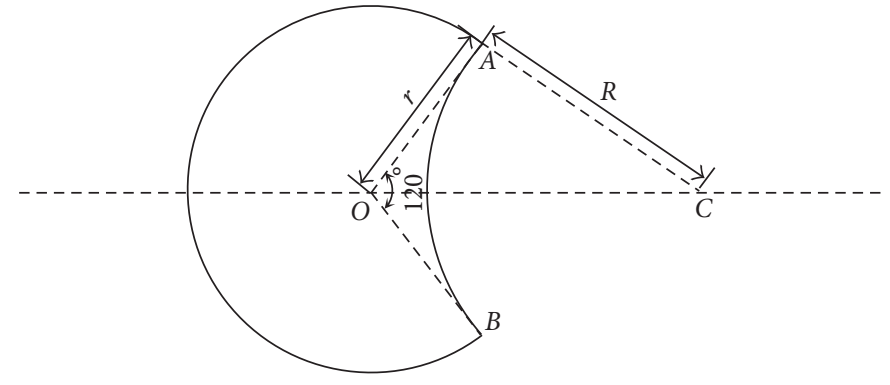

(b) Concave- $120^{\circ}$

Figure 2: The profiles of convex- $120^{\circ}$ and concave- $120^{\circ}$ structures.

TABLE 1: Material properties of the asymmetric structure.

\begin{tabular}{lcccccc}
\hline Part & Material & Density $\left(\mathrm{g} / \mathrm{cm}^{3}\right)$ & Yield stress $(\mathrm{MPa})$ & Young's modulus $(\mathrm{GPa})$ & Poisson's ratio & Shear modulus $(\mathrm{GPa})$ \\
\hline Charge & TNT & 1.64 & & & & \\
Endplate & LY12 Aluminum & 2.78 & 230 & 70 & 0.29 & 27.1 \\
Shell & 1020 Steel & 7.85 & 275 & 210 & 0.29 & 81.4 \\
\hline
\end{tabular}

the radius of another piece of circular arc is $R$, as shown in Figure 2. The two pieces of circular arc of the asymmetric shell were peripherally welded. Moreover, the endplates and asymmetric shell were connected by screws. The nominal diameter and height of TNT charge are $100 \mathrm{~mm}$ and $80 \mathrm{~mm}$, respectively. The upper and lower endplates have the same material and dimensions, with a diameter of $109.6 \mathrm{~mm}$ and a height of $10 \mathrm{~mm}$. In order to place the booster pellet, a cylindrical cave was punched in the circle center of the upper endplate. The inner diameter, thickness, and height of the asymmetric shell are $100 \mathrm{~mm}, 4.8 \mathrm{~mm}$, and $80 \mathrm{~mm}$, respectively. The concave and convex regions were made pregrooves by using the wire cutting process to control the fracture locations and obtain uniform fragments. The characteristic dimensions of the pregrooves are $4.8 \mathrm{~mm}$ in width, $3.8 \mathrm{~mm}$ in depth (along the axial direction), and $4.8 \mathrm{~mm}$ in depth (along the circumferential direction). Because the radius of the concave and convex profile is $100 \mathrm{~mm}$, there are 306 pieces of semiprefabricated fragments for the concave and convex regions.
2.2. Experimental Setup. The experimental setups of the asymmetric structure are shown in Figure 3. The experimental specimen was surrounded by the target plates. The target plates, with a width of $1 \mathrm{~m}$, a height of $2.5 \mathrm{~m}$, and a thickness of $0.3 \mathrm{~mm}$, were used to record the spatial distribution of fragments. Each plate made of steel was divided into several square regions $(25 \mathrm{~cm} \times 25 \mathrm{~cm})$ by gridlines. The distance between experimental specimen and target plates is $3.5 \mathrm{~m}$, so the azimuth angle of each square region is $4.09^{\circ}$. The TNT charge was initiated by electric detonator in the center of upper endplate. Meanwhile, the high speed photography and the oscilloscope were used to measure the arrival time of fragments. For the following analysis, it is assumed that the direction of $\overrightarrow{\mathrm{CO}}$ (corresponding to concave- $120^{\circ}$ ) or $\overrightarrow{\mathrm{OC}}$ (corresponding to convex $-120^{\circ}$ ) is the $0^{\circ}$ azimuth angle direction. In addition, it is defined that the azimuth angle is positive along the clockwise direction and negative along the counterclockwise direction. 


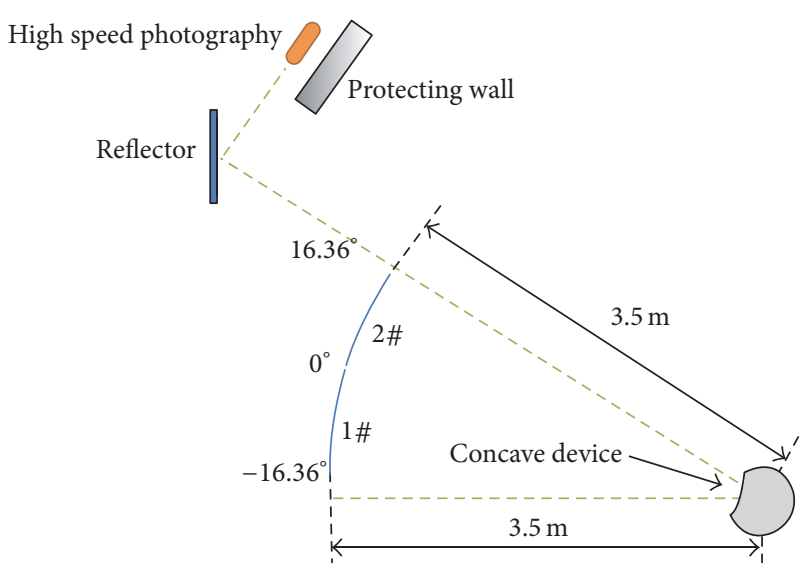

(a) The schematic diagram of concave- $120^{\circ}$

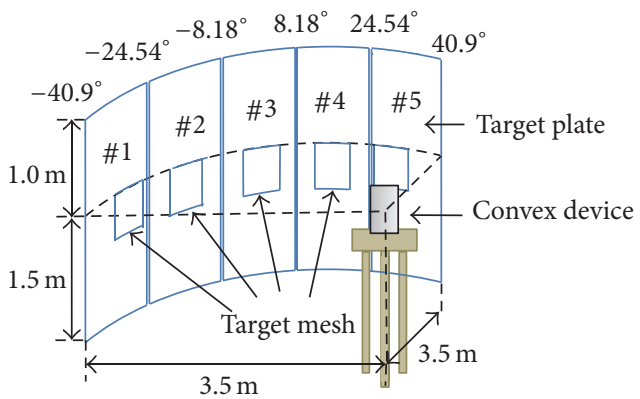

(c) The schematic diagram of convex- $120^{\circ}$

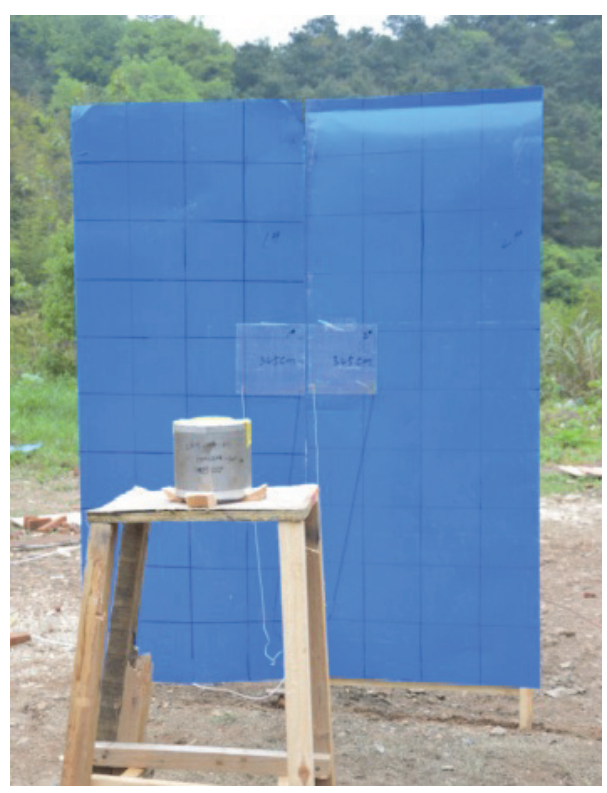

(b) The experimental layout of concave- $120^{\circ}$

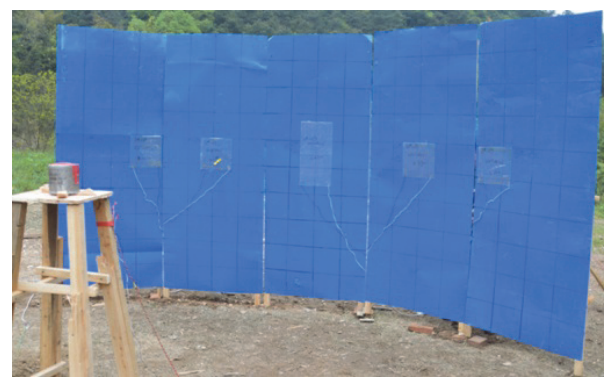

(d) The experimental layout of convex- $120^{\circ}$

FIGURE 3: Experimental setups of the asymmetric structures.

\subsection{Experimental Results}

2.3.1. Results on the Spatial Distribution of Fragments. The positions of fragments impacting on the target plate, corresponding to the concave specimen, are shown in Figure 4(a), and the figure indicates that the fragments formed a concentrated zone in the target direction. Because of some errors in the assembly process and site layout, the concentrated zone shifted to the right with approximately $2^{\circ}$. The number of fragments on the target plate was counted, as shown in Figure 4(b). The counted number is 285 and the total number of fragments is 306 , so the proportion is $93.1 \%$. The fragments were mainly distributed in the $-8.18^{\circ} \sim 12.27^{\circ}$ azimuth angle range, which were concentrated in the middle and sparse in both sides.

In the same way, the situation of fragments impacting on the target plate, corresponding to the convex specimen, as shown in Figure 4(c), and the fragments were counted as shown in Figure 4(d). The number of fragments obtained from the target plate is 293 , so the proportion is $95.7 \%$. The fragments were mainly distributed in the $-28.63^{\circ} \sim 28.63^{\circ}$ azimuth angle range, which were relatively uniform in the azimuth angle range.

2.3.2. Results on the Fragment Velocity. The high speed photography and oscilloscope were used to record the arrival time of fragments. Then, the average velocity of fragments can be calculated, and the initial velocity of fragments can also be derived according to the aerodynamic theory.

For the experiment of concave specimen, the high speed photography record under different moments is shown in Figures 5(a), 5(b), and 5(c), respectively. There were two target meshes which were used to measure the velocity, while only the \#2 target mesh captured the signal in the experiment. Therefore, the high speed photography record was mainly used to calculate the average velocity of fragments. The average velocity and initial velocity of concave structure obtained from the high speed photography and oscilloscope are listed in Table 2. Similarly, the average velocity and initial velocity of convex structure obtained from the high speed 


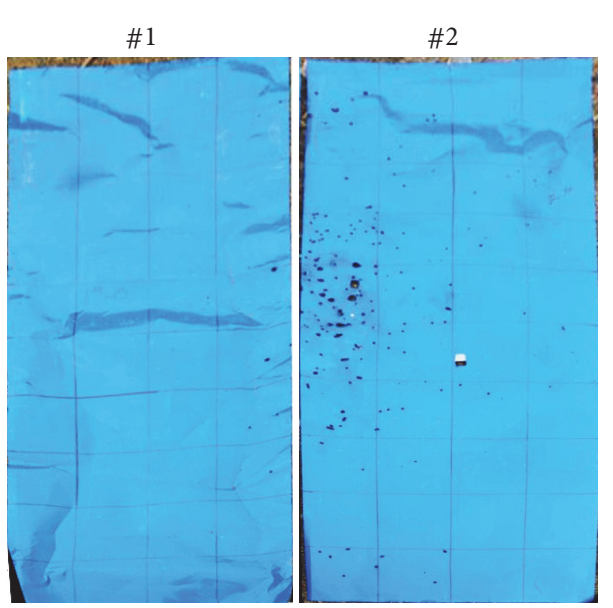

(a) Experimental photograph (concave- $120^{\circ}$ )

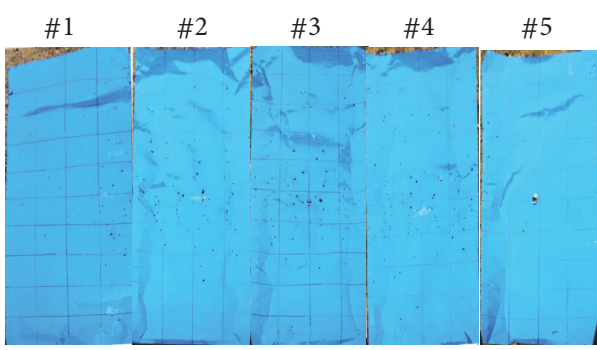

(c) Experimental photograph (convex- $120^{\circ}$ )

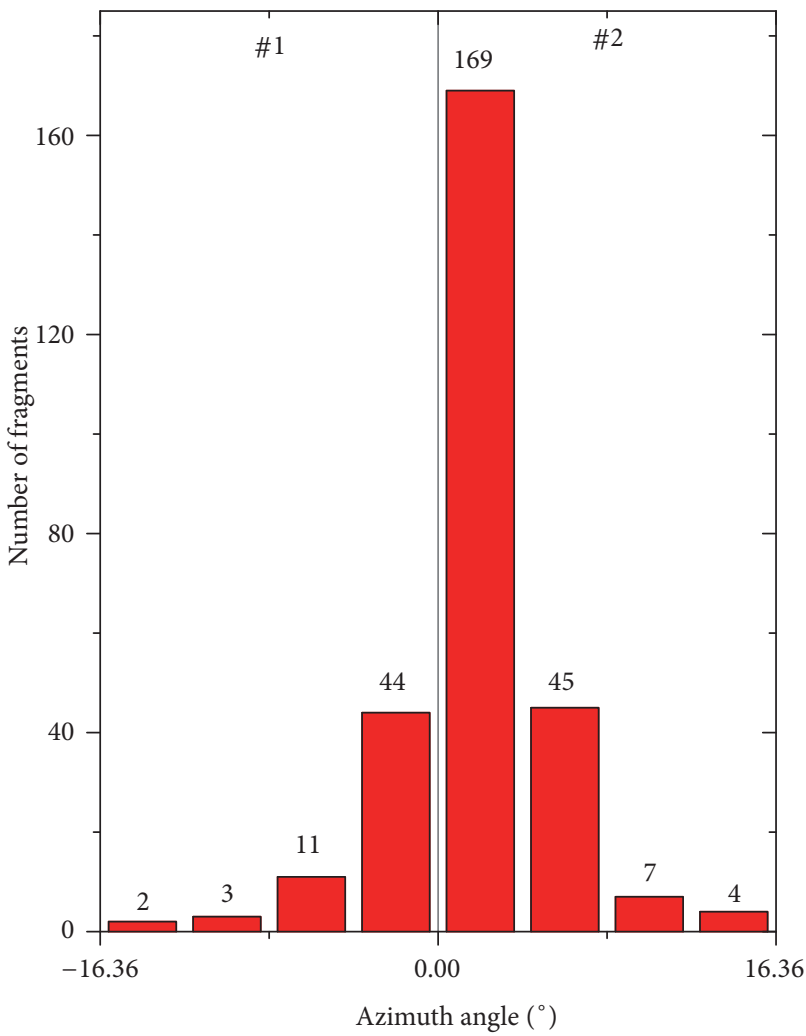

(b) The statistics result of fragment (concave- $120^{\circ}$ )

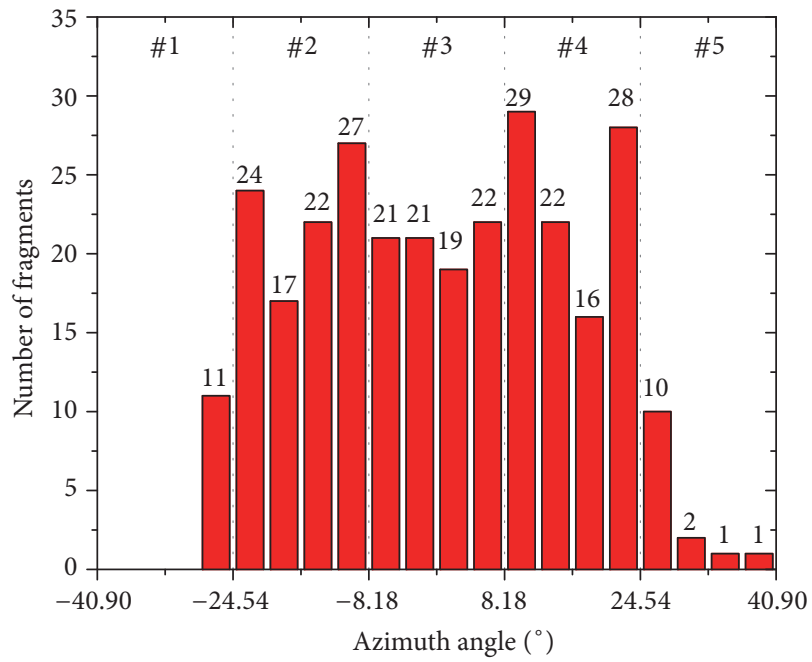

(d) The statistics result of fragment (convex- $120^{\circ}$ )

FIGURE 4: The experimental photograph and statistics of the asymmetric structures.

TABLE 2: Fragment velocity test results of concave- $120^{\circ}$ experiment.

\begin{tabular}{lccccccc}
\hline \multirow{2}{*}{$\begin{array}{l}\text { Target plate } \\
\text { number }\end{array}$} & \multirow{2}{*}{ Distance $(\mathrm{m})$} & \multicolumn{3}{c}{ Test results from oscilloscope $(\mathrm{m} / \mathrm{s})$} & \multicolumn{3}{c}{ Test results from high speed photography $(\mathrm{m} / \mathrm{s})$} \\
& & $\Delta t(\mathrm{~ms})$ & $\bar{v}(\mathrm{~m} / \mathrm{s})$ & $v_{0}(\mathrm{~m} / \mathrm{s})$ & $\Delta t(\mathrm{~ms})$ & $\bar{v}(\mathrm{~m} / \mathrm{s})$ & $v_{0}(\mathrm{~m} / \mathrm{s})$ \\
\hline$\# 1$ & 3.45 & \multicolumn{3}{c}{ No signal } & 2.08 & 1656 & 1722 \\
$\# 2$ & 3.45 & 2.0 & 1725 & 1799 & 1.92 & 1790 & 1861 \\
\hline
\end{tabular}




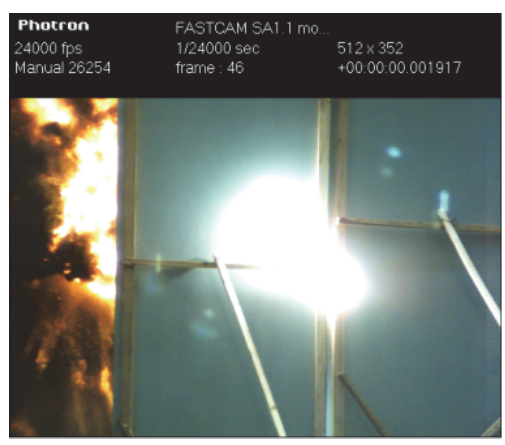

(a) $1.917 \mathrm{~ms}$

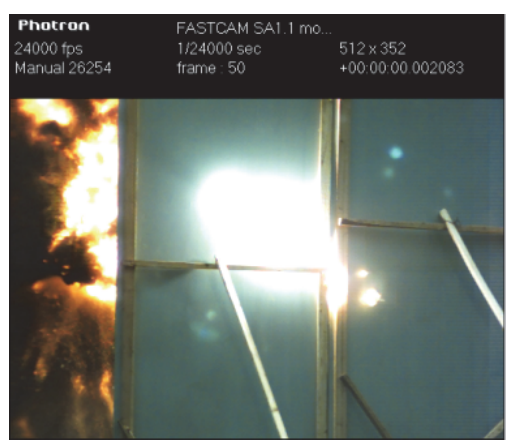

(b) $2.083 \mathrm{~ms}$

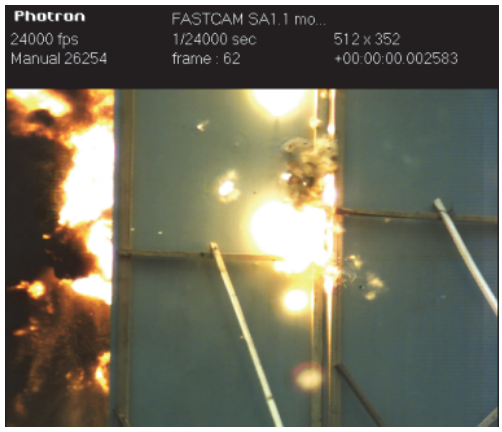

(c) $2.583 \mathrm{~ms}$

FIGURE 5: The high speed photography record of the concave- $120^{\circ}$ experiment.
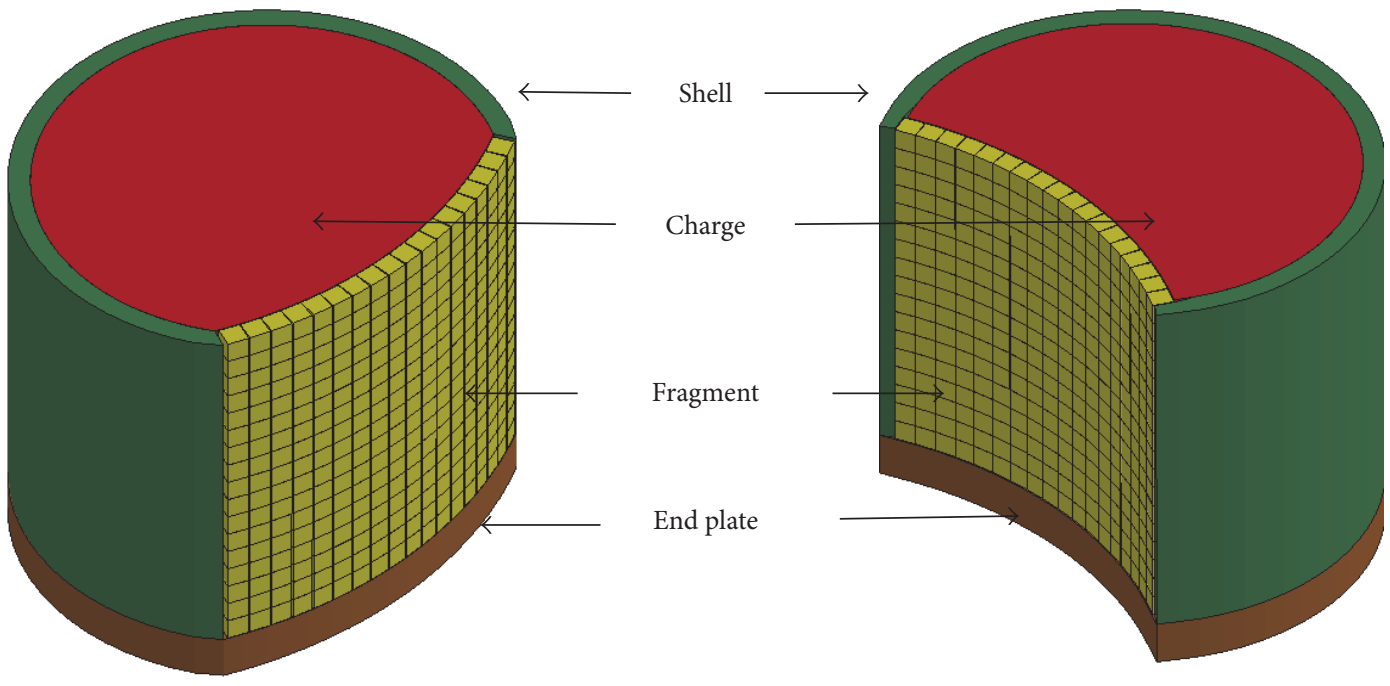

FIgURE 6: The FE model of concave- $120^{\circ}$ and convex- $120^{\circ}$.

TABLE 3: Fragment velocity test results of convex $-120^{\circ}$ experiment.

\begin{tabular}{lcccccc}
\hline \multirow{2}{*}{$\begin{array}{l}\text { Target plate } \\
\text { number }\end{array}$} & \multirow{2}{*}{ Distance $(\mathrm{m})$} & \multicolumn{2}{c}{ Test results from oscilloscope $(\mathrm{m} / \mathrm{s})$} & \multicolumn{3}{c}{ Test results from high speed photography $(\mathrm{m} / \mathrm{s})$} \\
& & $\Delta t(\mathrm{~ms})$ & $\bar{v}(\mathrm{~m} / \mathrm{s})$ & $v_{0}(\mathrm{~m} / \mathrm{s})$ & $\Delta t(\mathrm{~ms})$ & $\bar{v}(\mathrm{~m} / \mathrm{s})$ \\
\hline$\# 1$ & 3.42 & 2.33 & 1468 & 1533 & 2.44 & 1399 \\
$\# 2$ & 3.34 & No signal & & & 2.00 & 1398 \\
$\# 3$ & 3.39 & 1.87 & 1813 & 1893 & 1.83 & 1849 \\
$\# 4$ & 3.39 & 2.26 & 1500 & 1567 & 2.00 & 1695 \\
$\# 5$ & 3.39 & 2.31 & 1468 & 1533 & 2.38 & 1936 \\
\hline
\end{tabular}

photography and oscilloscope are listed in Table 3. The two tables indicate that the results of high speed photography coincide well with the results of oscilloscope.

\section{Simulation}

The numerical simulations were conducted by using LSDYNA (version 970), corresponding to the above experiments. LS-DYNA is a useful and powerful tool for solving the finite element problems, especially in the explosion, large deformation, high speed penetration, and so forth.

\subsection{FE Model}

3.1.1. Modeling Geometry. Based on the experiments, the models were built, as shown in Figure 6. In order to show the internal structure of the specimens, the upper endplate is not shown in Figure 6. For the convenience of modeling, 


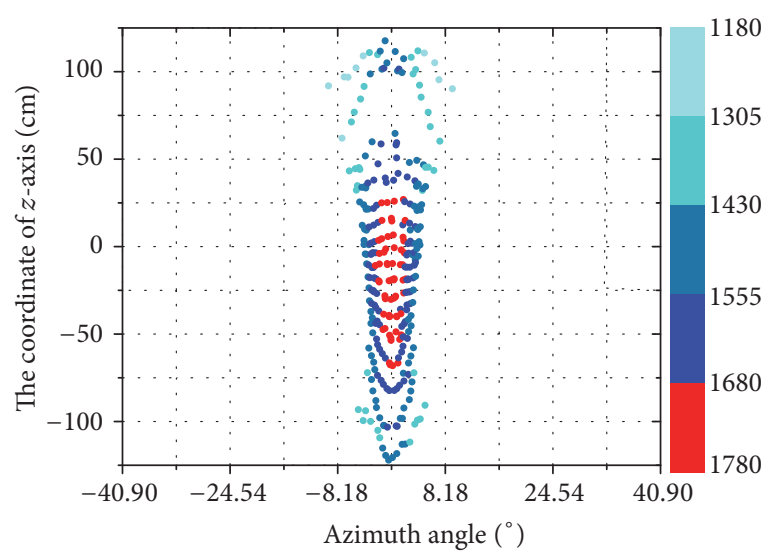

(a) Concave $-120^{\circ}$

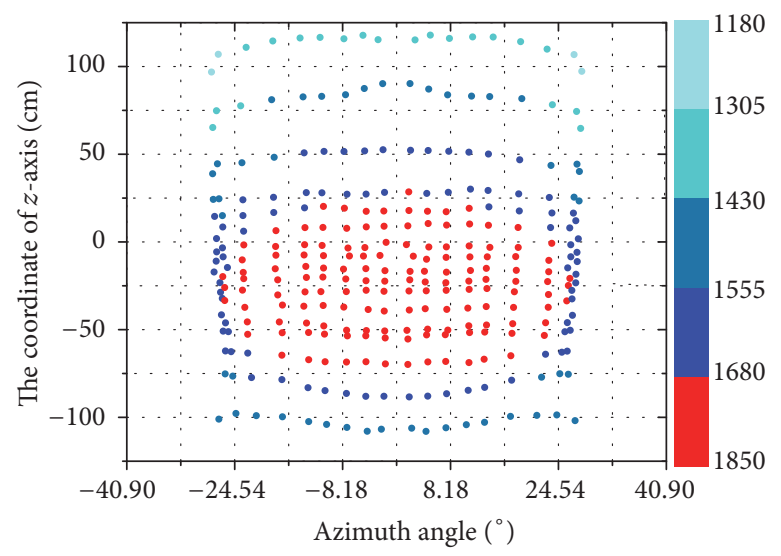

(b) Convex- $120^{\circ}$

FIgURE 7: The distributions of fragments from the simulation.

all of the semiprefabricated fragments were regarded as fullprefabricated fragments.

The entire model was modeled with solid elements, and the algorithm was ALE (Arbitrary Lagrange Euler). The ALE algorithm combines the characteristics of Euler algorithm and Lagrange Algorithm, so it is very suitable to analyze the fluid-solid coupling problems. Based on the requirements of the ALE algorithm, the whole model was defined as two parts, namely, the Euler part and the Lagrange part. The air and TNT charge were defined as Euler part, while the two endplates, shell, and fragment were defined as Lagrange part. The initiation point was located in the center of the upper surface of explosive.

3.1.2. Modeling Material. The selection of material model and equation of state is very important to the accuracy of numerical simulation. The material model and equation of state were introduced as follows in detail.

The high explosive material model ( ${ }^{*} \mathrm{MAT}_{-} \mathrm{HIGH}_{-}$ EXPLOSIVE_BURN) and the Jones-Wilkins-Lee equation of state (EOS_JWL) were used to describe the material property of TNT charge. The JWL equation of state gives the relation between the pressure of detonation products and various parameters, which is expressed as

$$
\begin{aligned}
P= & A\left(1-\frac{\omega}{R_{1} V}\right) e^{-R_{1} V}+B\left(1-\frac{\omega}{R_{2} V}\right) e^{-R_{2} V} \\
& +\frac{\omega E_{0}}{V}
\end{aligned}
$$

where $P$ is the detonation pressure, $V$ is the initial relative volume, $E_{0}$ is the initial specific internal energy, and $A, B, R_{1}, R_{2}$, $\omega$ are material constants. The specific parameters are listed in Table 4.

The material type $9\left({ }^{*}\right.$ MAT_NULL $)$ in LS-DYNA incorporating the linear polynomial equation of state (EOS_LINEAR_POLYNOMIAL) was used to describe the material property of air. The linear polynomial equation of state is a linear function of internal energy; the pressure is expressed as

$$
\begin{aligned}
P= & C_{0}+C_{1} \mu+C_{2} \mu^{2}+C_{3} \mu^{3} \\
& +\left(C_{4}+C_{5} \mu+C_{6} \mu^{2}\right) E_{0} .
\end{aligned}
$$

If $C_{0}=C_{1}=C_{2}=C_{3}=C_{6}=0$, (2) can describe the material property of air very well. The specific parameters of air are also listed in Table 4.

Other components, including the shell, endplates, and fragments, were modeled by the material type $10\left({ }^{*} \mathrm{MAT}_{-}\right.$ ELASTIC_PLASTIC_HYDRO_SPALL) and the Gruneisen equation of state (EOS_GRUNEISEN) in LS-DYNA.

3.2. Comparisons between Simulation and Experiment. The spatial distributions of fragments in the target plates were illustrated in Figure 7; the fragment velocities were distinguished by different colors. It is seen from the diagram that the fragments have a significant concentrated effect for the concave structure, and the fragments mainly distributed in the $-8.18^{\circ} \sim 8.18^{\circ}$ azimuth angle range. However, the fragments of convex structure distributed uniformly in the $-26.58^{\circ} \sim 26.58^{\circ}$ azimuth angle range.

For the concave $-120^{\circ}$ structure, the experimental data and numerical simulation data are shown in Figure 8. It is seen from Figure 8(a) that the experimental results decreased by about $6 \%$ when compared with the simulation results. Because the fragments distributed in a smaller angle range, it was difficult to confirm the specific number of fragments in the positions with big holes, which eventually led to that the statistics was lower than the normal condition. Based on Figure 8(a), the statistics of fragments from the experiment is smaller, so the average velocity of fragment from the experiment is higher than the simulation as shown in Figure 8(b). In addition, the concentrated zone shifted to the right with approximately $2^{\circ}$. There are almost no fragments in the $4.09^{\circ}$ azimuth angle, as shown in Figure 4(a), so the fragment velocity from the experiment is vacant in the $4.09^{\circ}$ azimuth angle. Nonetheless, the fragment velocity in the 


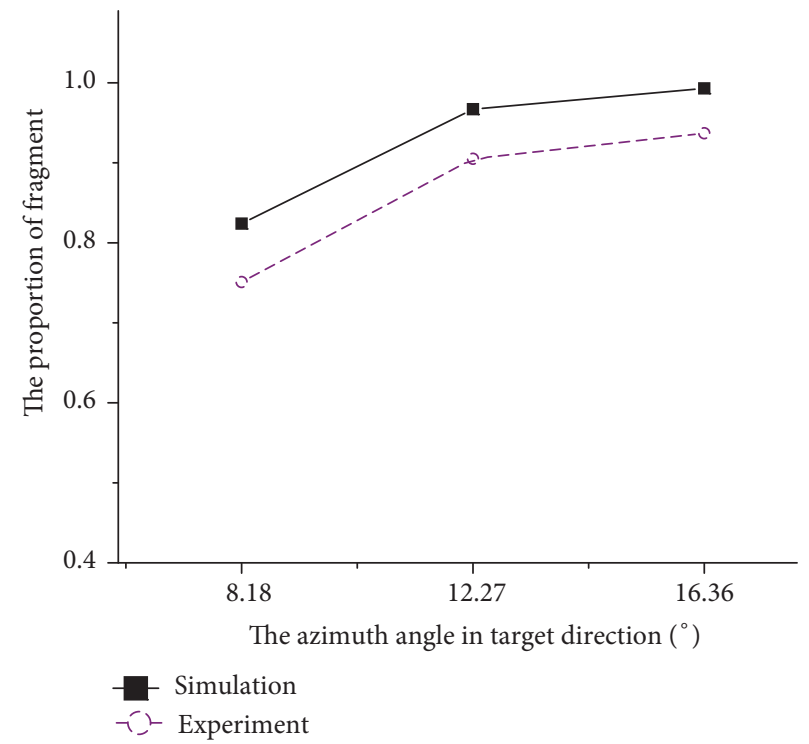

(a) The proportion of fragment (concave- $120^{\circ}$ )

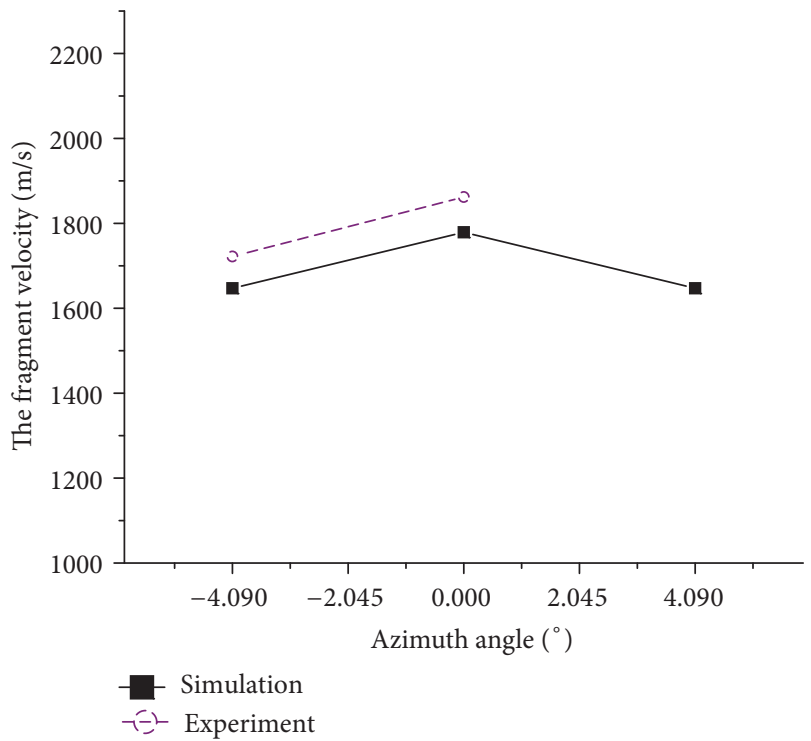

(b) The fragment velocity (concave- $120^{\circ}$ )

Figure 8: The simulation data and experiment data of concave- $120^{\circ}$.

TABLE 4: Input data in the numerical simulation (unit $=\mathrm{cm}, \mathrm{g}$, and $\mu \mathrm{s}$ ).

\begin{tabular}{|c|c|c|c|c|c|c|c|c|}
\hline \multirow{2}{*}{ Part } & \multirow[t]{2}{*}{ Material } & \multicolumn{7}{|c|}{ LS-DYNA material type, material property, and EOS input data } \\
\hline & & ${ }^{*}$ MAT_H & LOSIVE_H & & & & & \\
\hline \multirow{5}{*}{ Charge } & \multirow{5}{*}{ TNT } & RO & $\mathrm{D}$ & PCJ & & & & \\
\hline & & 1.640 & 0.693 & 0.27 & & & & \\
\hline & & ${ }^{*}$ EOS_JW & & & & & & \\
\hline & & A & $B$ & $R 1$ & $R 2$ & OMEG & E0 & $V 0$ \\
\hline & & 3.74 & $3.23 E-2$ & 4.15 & 0.95 & 0.3 & 0.09 & 1.0 \\
\hline \multirow{6}{*}{ Air } & \multirow{6}{*}{ Air } & ${ }^{*}$ MAT_N & & & & & & \\
\hline & & RO & & & & & & \\
\hline & & $1.293 E-$ & & & & & & \\
\hline & & ${ }^{*}$ EOS_LII & LYNOML & & & & & \\
\hline & & $C 4$ & $C 5$ & E0 & $V 0$ & & & \\
\hline & & 0.4 & 0.4 & $2.5 E-6$ & 1.0 & & & \\
\hline \multirow{6}{*}{ Shell } & \multirow{6}{*}{ Steel } & ${ }^{*}$ MAT_EI & LASTIC_I & D_SPALL & & & & \\
\hline & & RO & G & SIGY & $\mathrm{EH}$ & $\mathrm{PC}$ & FS & \\
\hline & & 7.85 & 0.618 & $0.724 E-2$ & 0.28 & -7.03 & 0.36 & \\
\hline & & ${ }^{*}$ EOS_GR & & & & & & \\
\hline & & $C$ & $S 1$ & GMAO & & & & \\
\hline & & 0.3574 & 1.92 & 1.69 & & & & \\
\hline \multirow{6}{*}{ Endplate } & \multirow{6}{*}{ Aluminum } & ${ }^{*}$ MAT_EI & LASTIC_I & D_SPALL & & & & \\
\hline & & RO & G & SIGY & $\mathrm{EH}$ & $\mathrm{PC}$ & FS & \\
\hline & & 2.73 & 0.265 & $2.95 E-03$ & $8.384 E-3$ & -9.0 & 0.4 & \\
\hline & & ${ }^{*}$ EOS_GR & & & & & & \\
\hline & & $C$ & $S 1$ & GMAO & & & & \\
\hline & & 0.5328 & 1.338 & 2.0 & & & & \\
\hline
\end{tabular}




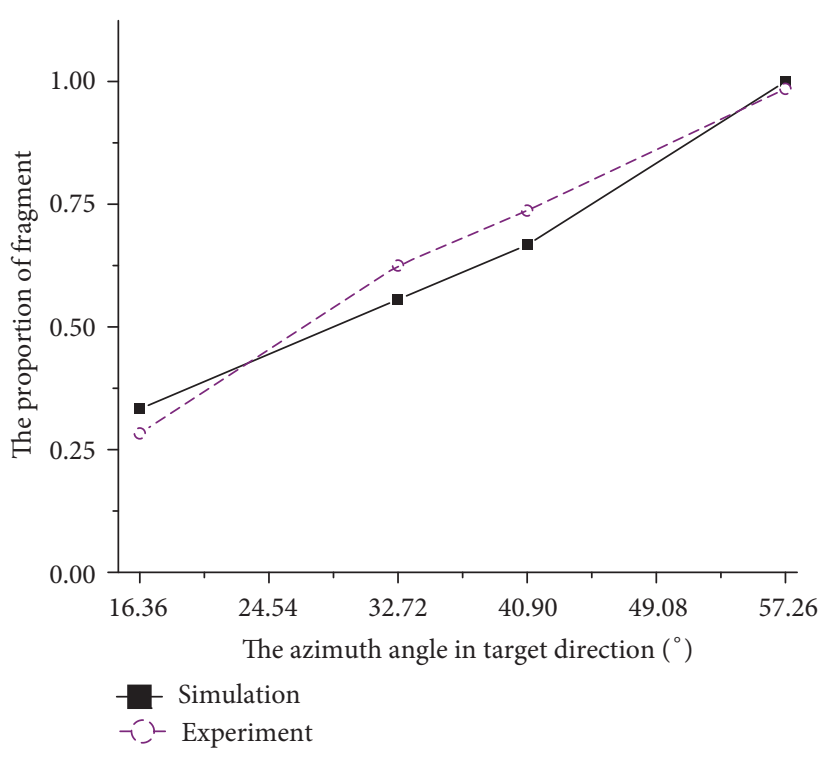

(a) The proportion of fragment (convex- $120^{\circ}$ )

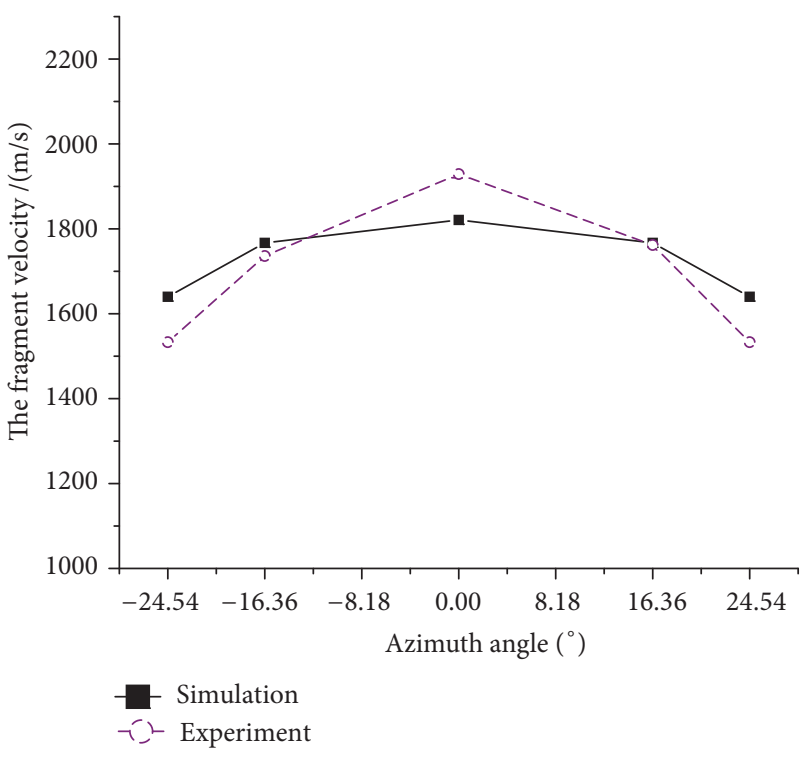

(b) The fragment velocity (convex- $120^{\circ}$ )

FIgURE 9: The simulation data and experiment data of convex-120 .

$4.09^{\circ}$ azimuth angle should be the same with the fragment velocity in the $-4.09^{\circ}$ azimuth angle, which is $1722 \mathrm{~m} / \mathrm{s}$.

The results of experimental data and simulation data are shown in Figure 9, and the simulation coincides well with the experiment, as shown in Figure 9(a). The fragment velocities from the experiment and the simulation have the same change trend, and the error is less than $5 \%$, as shown in Figure 9(b). The maximum fragment velocity of convex- $120^{\circ}$ is higher than that of concave- $120^{\circ}$, because the charge mass of convex $-120^{\circ}$ is larger than the concave- $120^{\circ}$.

Through the comprehensive analysis of the results, and taking into account the experimental errors, the numerical simulation can accurately reflect the dispersion rules of fragments.

\section{The Dispersion Rule of Fragments}

4.1. The Dispersion Rule for the Concave Structure. In the simulations of concave- $120^{\circ}$ structure, the fragments affected each other in the dispersion process, which generated spall of fragment and wasted the fragment energy. This phenomenon will seriously affect the damage effectiveness of fragment, so it is necessary to study the relationship between $R$ and $r$ to avoid the interaction among fragments.

In order to find a suitable proportion relationship, several kinds of simulations were carried out; namely, $R / r=4,5$, $6,7,8$ ( $R$ is the concave profile radius and $r$ is the charge radius). The dispersion process under different moments is shown in Figure 10, under the condition of $R / r=6$. The simulation results of fragments impacting on the target plates are illustrated in Figure 11, corresponding to the five groups of simulations, respectively.

It is seen from Figures 10 and 11 that the fragments were gradually concentrated in the middle of target plate with the increase of $R / r$, under the condition of $R<6 r$. However, the fragments were divergent in the dispersion process, under the condition of $R>6 r$. So the optimal curvature of the concave$120^{\circ}$ structure is $R=6 r$, and most of the fragments were concentrated in the target direction under this condition. The increase of projection angle and the loss of kinetic energy were avoided in the greatest extent, which enhanced the effectiveness of fragment.

The simulations of concave- $90^{\circ}$ and concave- $150^{\circ}$ were carried out and analyzed by the same method. The final results showed that the optimal curvatures of the concave- $90^{\circ}$ and concave- $150^{\circ}$ are $R=4 r$ and $R=6 r$.

Based on the above simulation analysis, it can be drawn that the optimal curvature changes from $4 r$ to $6 r$ when the central angle of concave structure changes from $90^{\circ}$ to $120^{\circ}$. However, the optimal curvature does not change significantly when the central angle is larger than $120^{\circ}$.

4.2. The Dispersion Rule for the Convex Structure. The experiment of convex- $120^{\circ}-2 r$ was carried out, and the experimental result reported that the fragments distributed uniformly in the circumferential direction, under the condition of $R=2 r$. In order to study the dispersion rule of fragments under different radius of curvature, four groups of numerical simulation were built; namely, $R / r=2,3,4,5$. The simulation results of fragments impacting on the target plates are illustrated in Figure 12, respectively.

The simulation results indicate that the fragments are uniformly distributed in the circumferential direction, under the condition of $R<3 r$. When the radius of curvature continues to increase, the shape of convex structure is more closer to the D-shape, and the distribution of fragments is more closer to the state which is dense in the middle and sparse on both sides. 


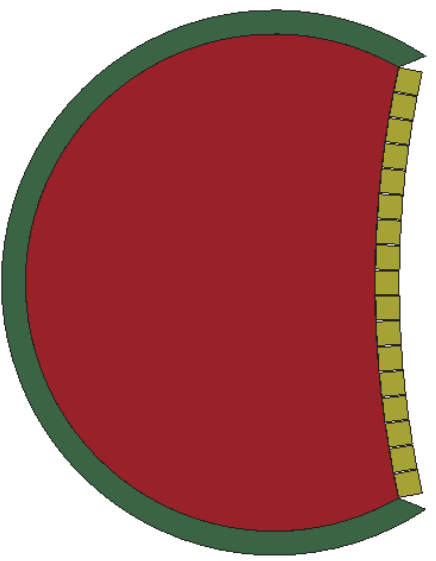

(a) $0 \mu \mathrm{s}$

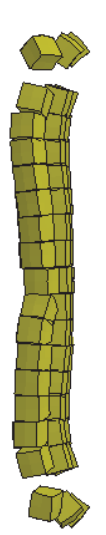

(b)

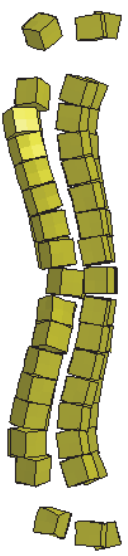

(c) $120 \mu \mathrm{s}$

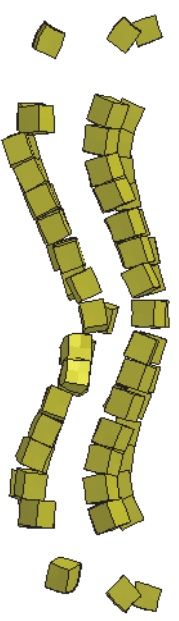

(d) $180 \mu \mathrm{s}$

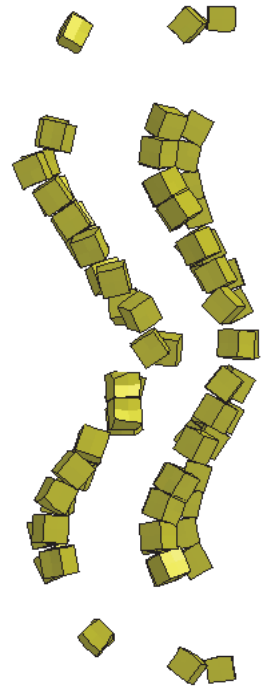

(e) $240 \mu \mathrm{s}$

Figure 10: The fragment dispersion process of concave- $120^{\circ}, 6 r$.

TABLE 5: The relationship between the curvature and fragment projection angle range.

\begin{tabular}{lc}
\hline$R / r$ & Projection angle range \\
\hline 1.15 & 46.4 \\
1.3 & 40.0 \\
1.5 & 35.6 \\
1.7 & 32.8 \\
1.85 & 30.6 \\
2.0 & 27.7 \\
3.0 & 24.0 \\
4.0 & 22.8 \\
5.0 & 21.9 \\
\hline
\end{tabular}

In the same way, several models with the radius of curvature ranging from $R=r$ to $R=5 r$ were conducted. Through the analysis of simulation results, the relationship between the radius of curvature and the fragment projection angle range is listed in Table 5. Based on the data from Table 5, the relation curve between the curvature and the fragment projection angle range was fitted, as shown in Figure 13, corresponding to the convex $-120^{\circ}$ structure. In Figure 13, the horizontal axis represents the ratio of the curvature and the charge radius; the vertical axis represents the azimuth angle of the outermost fragments.

In order to rapidly predict the projection angle in case of the curvature and the central angle of the convex profile were given, a fitting formula based on the data from Table 5 was obtained. The fitting formula was expressed as follows:

$$
\theta=A+B \alpha+C\left(\frac{R}{r}\right)+D \alpha^{2}+E\left(\frac{R}{r}\right)^{2}+F \alpha\left(\frac{R}{r}\right)
$$

TABLE 6: The specific value of the fitting parameters.

\begin{tabular}{lc}
\hline Fitting parameter & Value \\
\hline$A$ & 42.8298 \\
$B$ & $6.144 \cdot 10^{-3}$ \\
$C$ & -14.4233 \\
$D$ & $1.307 \cdot 10^{-3}$ \\
$E$ & 2.326 \\
$F$ & $-4.375 \cdot 10^{-2}$ \\
\hline
\end{tabular}

TABLE 7: The contrasts between the simulation and the formula.

\begin{tabular}{lcccc}
\hline \multirow{2}{*}{$R / r$} & \multicolumn{2}{c}{ Convex-90 } & \multicolumn{2}{c}{ Convex-150 } \\
& Simulation & Formula & Simulation & Formula \\
\hline 1.3 & 34 & 33.61 & 52.4 & 49.11 \\
1.7 & 29.06 & 28.93 & 42.41 & 43.29 \\
3.0 & 21 & 18.85 & 30.24 & 29.52 \\
5.0 & 16 & 18.7 & 22.04 & 23.67 \\
\hline
\end{tabular}

where $\theta$ is the azimuth angle of the outermost fragments, $\alpha$ is the central angle of the convex profile, $R / r$ is the ratio of the curvature and the charge radius, and $A, B, C, D, E, F$ are the fitting constants and the specific values of which are shown in Table 6.

A series of models under different conditions were constructed to verify the reliability and rationality of formula (3). The results from the formula and the simulation are listed in Table 7, which indicates that both of them coincide very well. Therefore, the fitting formula can rapidly predict the projection angle range of fragments about the convex structure. 


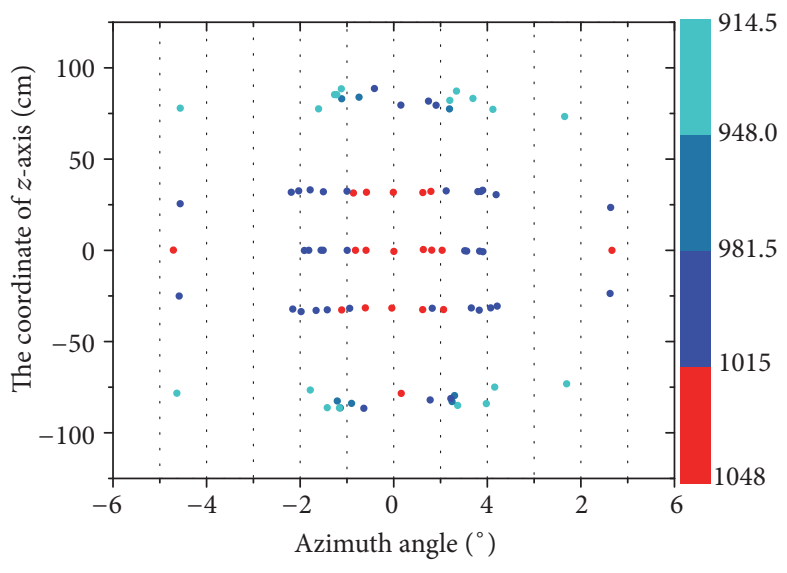

(a) Concave $-120^{\circ}-4 r$

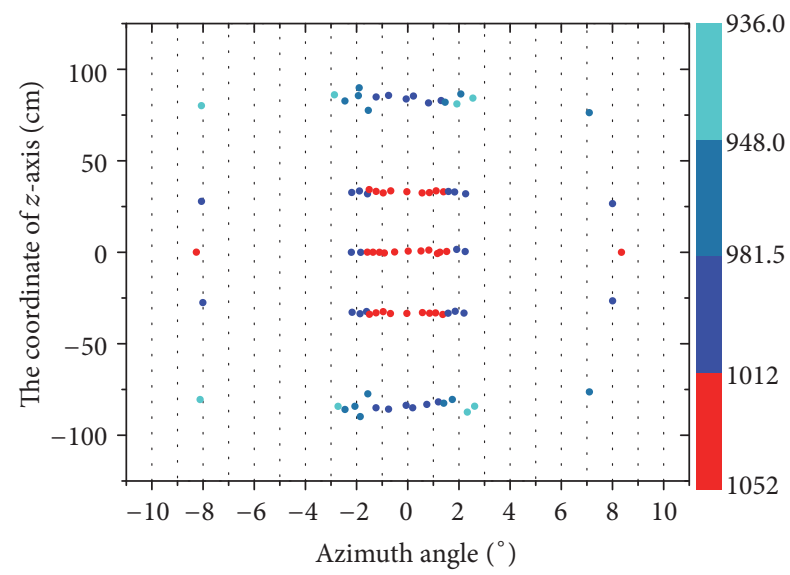

(c) Concave- $120^{\circ}-6 r$

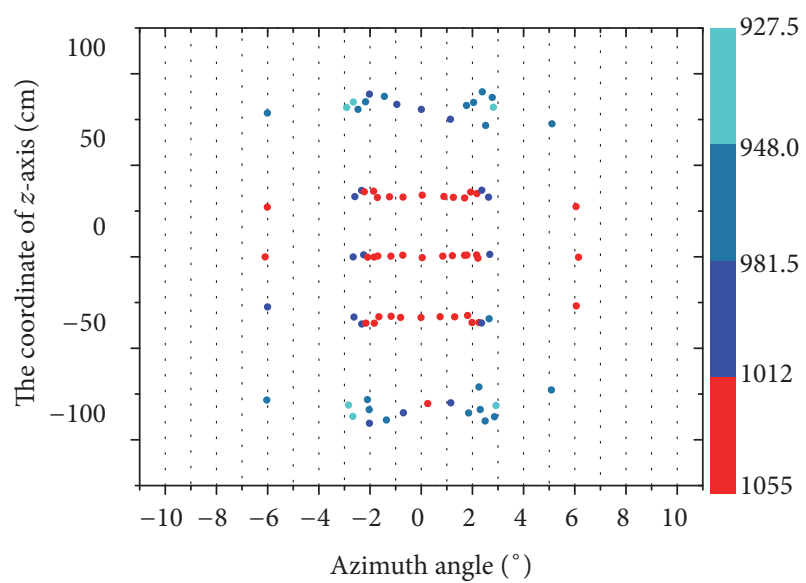

(b) Concave $-120^{\circ}-5 r$

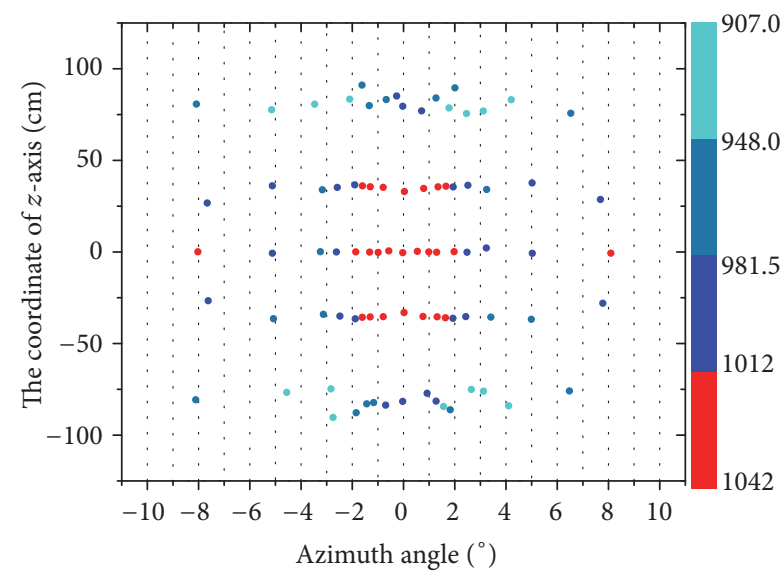

(d) Concave $-120^{\circ}-7 r$

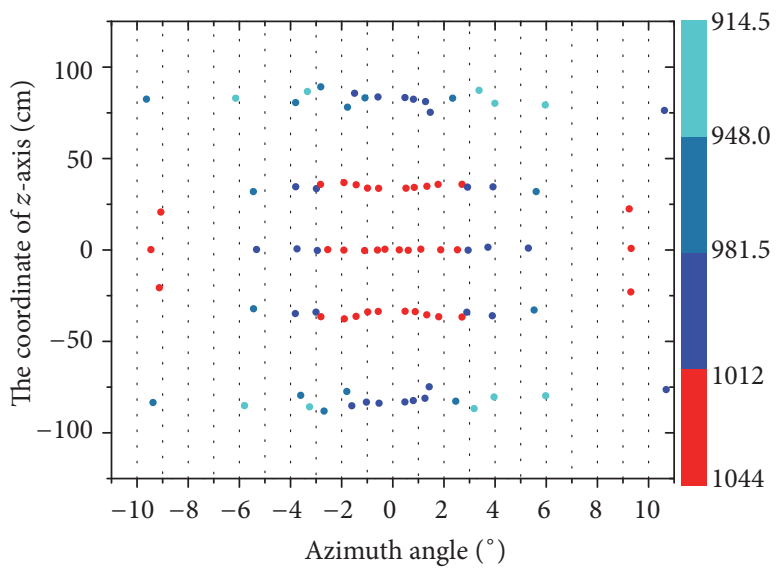

(e) Concave- $120^{\circ}-8 r$

FIGURE 11: The spatial distributions of fragment corresponding to concave structures.

\section{Conclusions}

This paper has carried out the experiment and numerical simulation about the concave- $120^{\circ}$ structure and convex $-120^{\circ}$ structure; the results indicated that the simulation results coincided well with the experimental results. For the concave structure, the optimal curvature changes from $4 r$ to $6 r$ when the central angle of concave structure changes from $90^{\circ}$ to $120^{\circ}$. However, the optimal curvature does not change significantly when the central angle is larger than $120^{\circ}$. For the convex $-120^{\circ}$, the fragments are uniformly distributed in the circumferential direction, under the condition of $R<$ $3 r$. The profile of convex structure is more closer to the Dshape, under the condition of $R>3 r$, and the distribution 


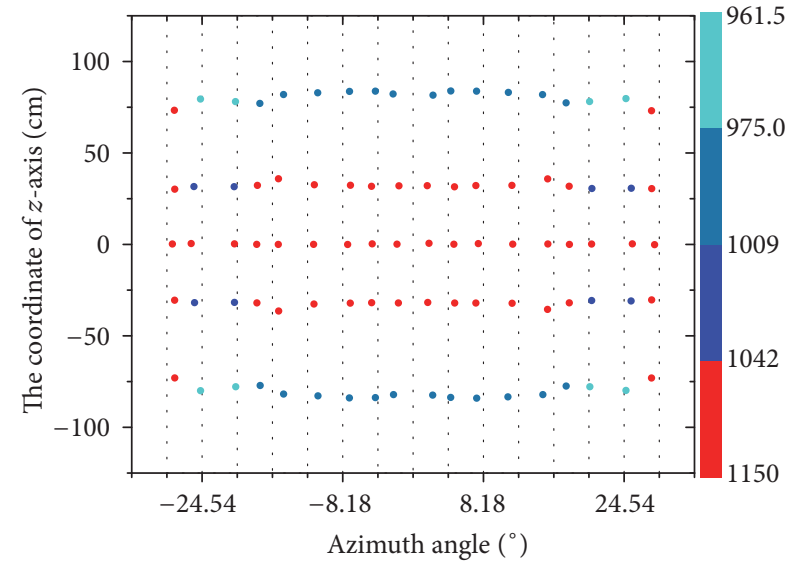

(a) Convex- $120^{\circ}-2 r$

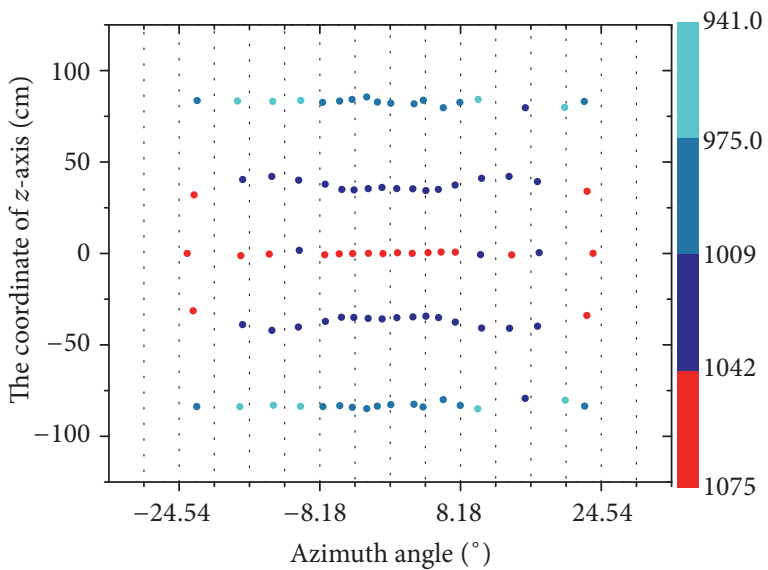

(c) Convex- $120^{\circ}-4 r$

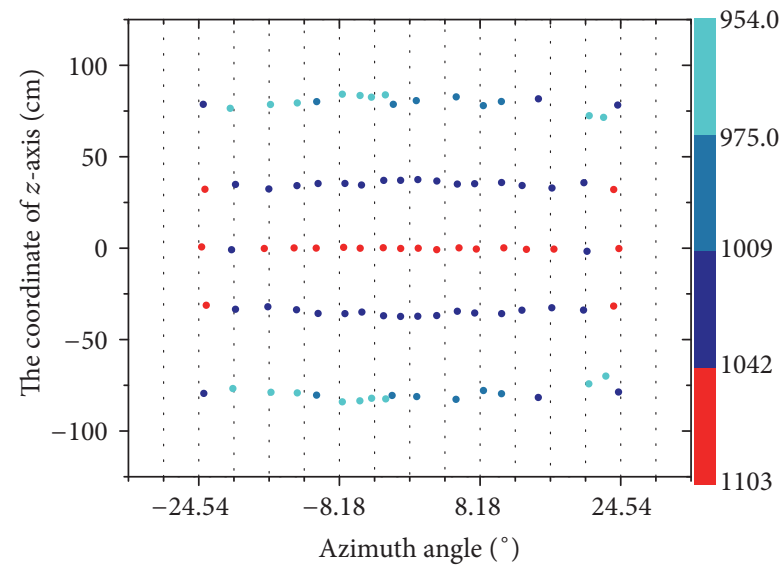

(b) Convex- $120^{\circ}-3 r$

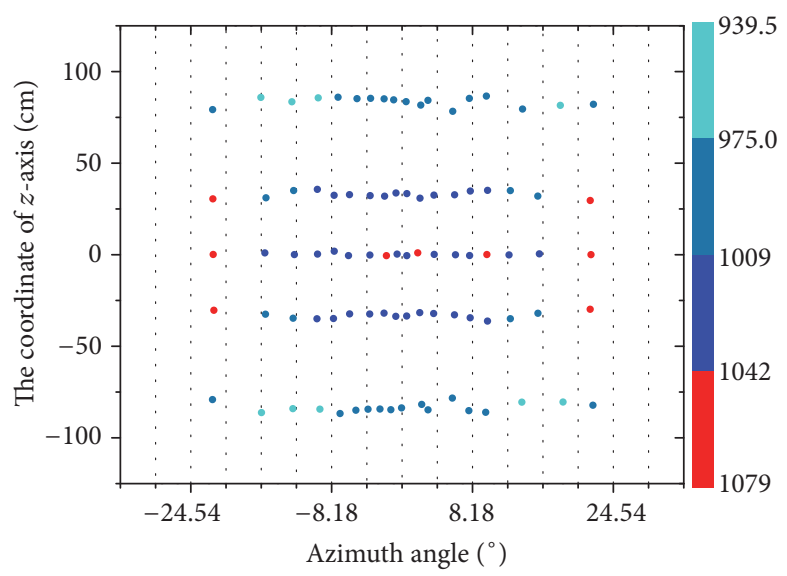

(d) Convex- $120^{\circ}-5 r$

FIGURE 12: The spatial distributions of fragment corresponding to convex structures.

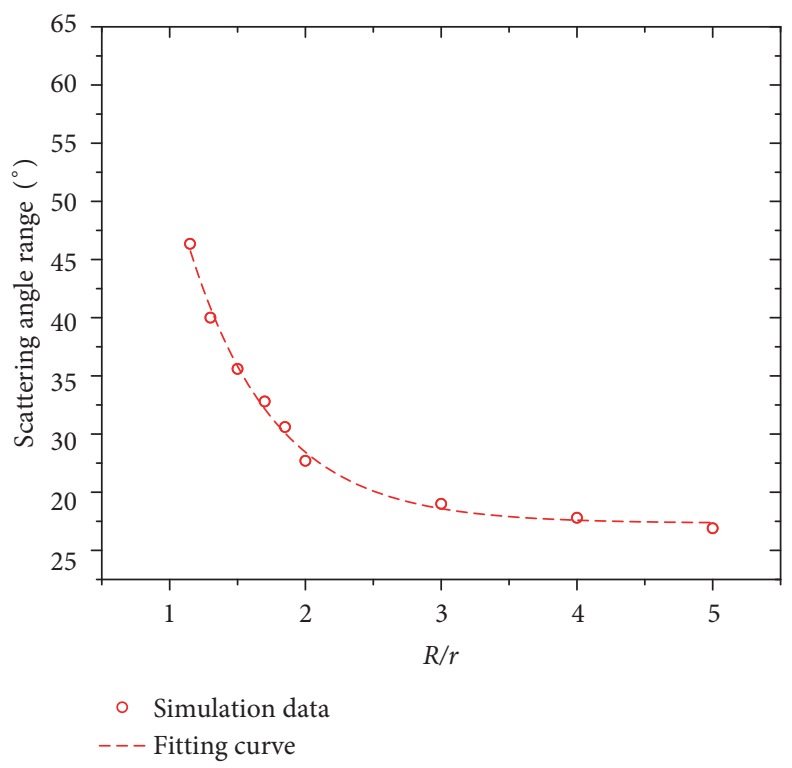

FIGURE 13: The fitting curve of curvature and projection angle range. 
of fragments is more closer to the state which is dense in the middle and sparse on both sides. In order to rapidly predict the projection angle range of the convex structure, a formula was fitted as follows:

$$
\theta=A+B \alpha+C\left(\frac{R}{r}\right)+D \alpha^{2}+E\left(\frac{R}{r}\right)^{2}+F \alpha\left(\frac{R}{r}\right)
$$

The formula was verified; the result indicated that it was reasonable and reliable.

\section{Competing Interests}

The authors declare that there is no conflict of interests regarding the publication of this paper.

\section{Acknowledgments}

This work is financially supported by the National Natural Science Foundation of China, under Grants nos. 11202237 and 11132012. The financial contributions are gratefully acknowledged.

\section{References}

[1] R. W. Gurney, The Initial Velocities of Fragments from Bombs, Shells and Grenades, BRL Report No. 405, 1943.

[2] G. I. Taylor, Analysis of the Explosion of a Long Cylindrical Bomb Detonated at One End, Cambridge University Press, Cambridge, UK, 1963.

[3] N. F. Mott, "Fragmentation of shell cases," Proceedings of the Royal Society of London, vol. 189, no. 1018, pp. 300-308, 1947.

[4] P. C. Chou, J. Carleone, W. J. Flis, R. D. Ciccarelli, and E. Hirsch, "Improved formulas for velocity, acceleration, and projection angle of explosively driven liners," Propellants, Explosives, Pyrotechnics, vol. 8, no. 6, pp. 175-183, 1983.

[5] P. J. König, "A correction for ejection angles of fragments from cylindrical wareheads," Propellants, Explosives, Pyrotechnics, vol. 12, no. 5, pp. 154-157, 1987.

[6] M. Held, "Diagnostics of the profile and rotational symmetry of a detonation Wave," Propellants, Explosives, Pyrotechnics, vol. 22, no. 2, pp. 93-96, 1997.

[7] M. Wang, F. Lu, X. Li, and L. Cao, "A formula for calculating the velocities of fragments from velocity enhanced warhead," Propellants, Explosives, Pyrotechnics, vol. 38, no. 2, pp. 232-237, 2013.

[8] K. D. Dhote, K. P. S. Murthy, K. M. Rajan, and M. M. Sucheendran, "Statistics of fragment dispersion by explosion in a fragment generator warhead," Central European Journal of Energetic Materials, vol. 13, no. 1, pp. 183-197, 2016.

[9] Y. P. Lian, X. Zhang, X. Zhou, S. Ma, and Y. L. Zhao, "Numerical simulation of explosively driven metal by material point method," International Journal of Impact Engineering, vol. 38, no. 4, pp. 238-246, 2011.

[10] G. Y. Huang, W. Li, and S. S. Feng, "Fragment velocity distribution of cylindrical rings under eccentric point initiation," Propellants, Explosives, Pyrotechnics, vol. 40, no. 2, pp. 215-220, 2015.

[11] L. Wang, F. Han, and F. Chen, "Fragment velocity distribution of velocity enhanced warhead under double symmetric initiations," Propellants, Explosives, Pyrotechnics, vol. 41, no. 4, pp. 672-681, 2016.
[12] K. D. Dhote, P. N. Verma, and K. P. S. Murthy, "Mitigation of fragment spall induced by explosive loading in high performance fragment generators," Journal of Battlefield Technology, vol. 17, no. 1, pp. 6-8, 2014.

[13] V. M. Gold, "Warhead Selectively Releasing Fragments of Varied Sizes and Shapes," US Patent 8061275, 2011.

[14] B.-L. Gong, F.-Y. Lu, X.-Y. Li, and Y.-L. Lin, "Study on fragment projection angle of d-shaped warhead," in Proceedings of the 25th International Symposium on Ballistics (ISB '10), pp. 728 735, Beijing, China, May 2010.

[15] M. Wang, F. Lu, X. Li, and L. Cao, "A new method to estimate the projection angles of fragments from a $\mathrm{D}$ shape configuration," Applied Mechanics and Materials, vol. 275-277, pp. 122-127, 2013. 


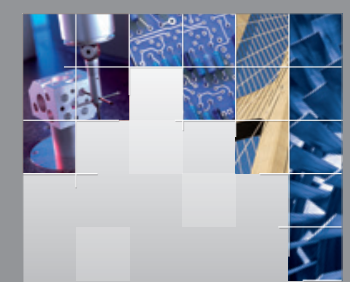

\section{Enfincering}
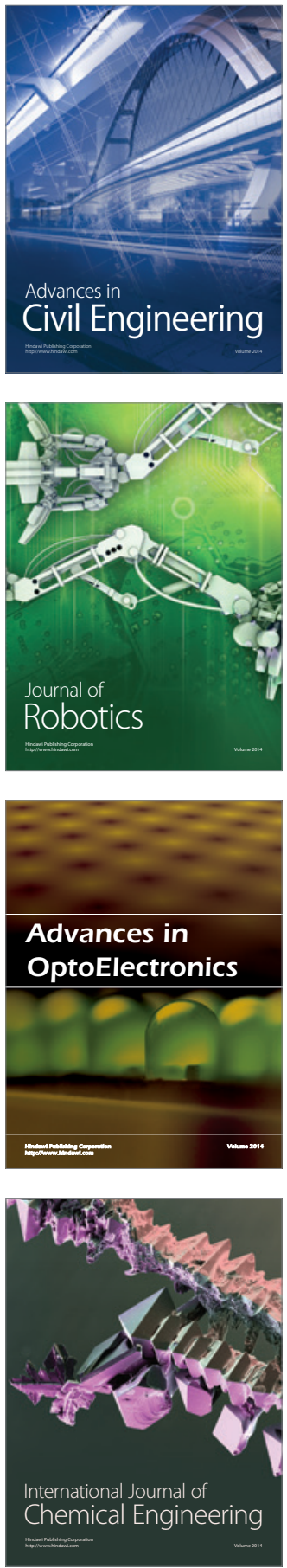

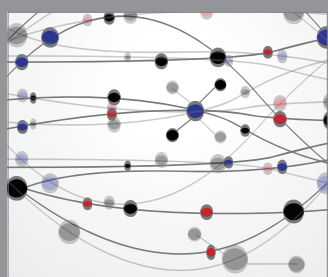

The Scientific World Journal

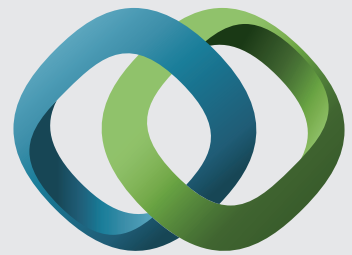

\section{Hindawi}

Submit your manuscripts at

https://www.hindawi.com
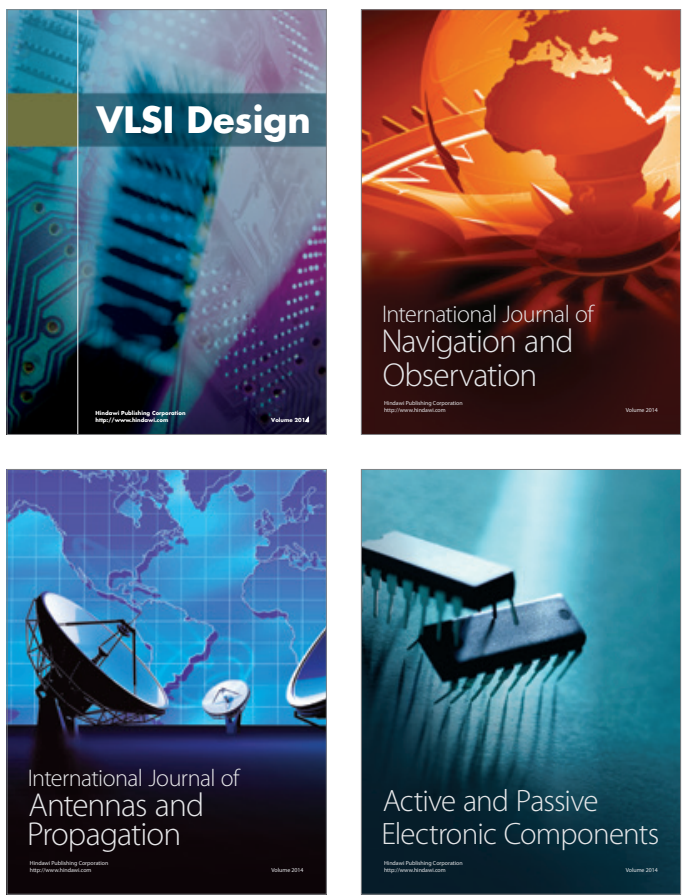
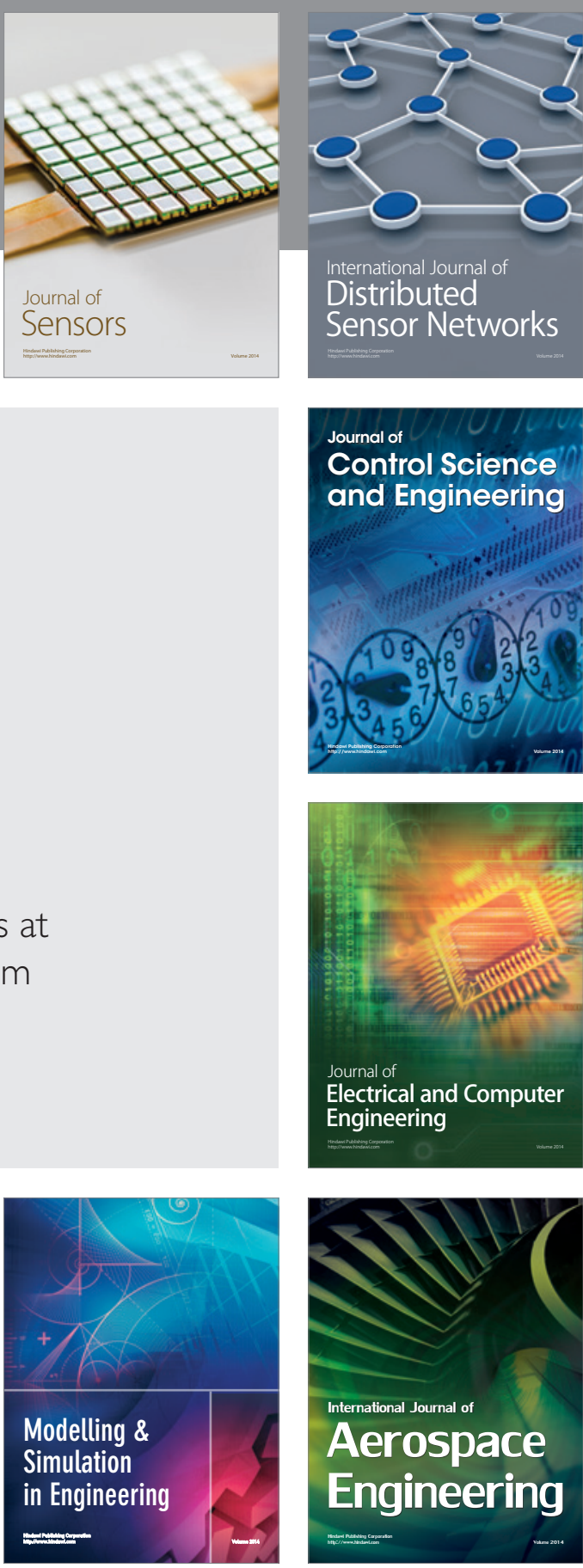

International Journal of

Distributed

Sensor Networks

$-$

Joumal of

Control Science

and Engineering
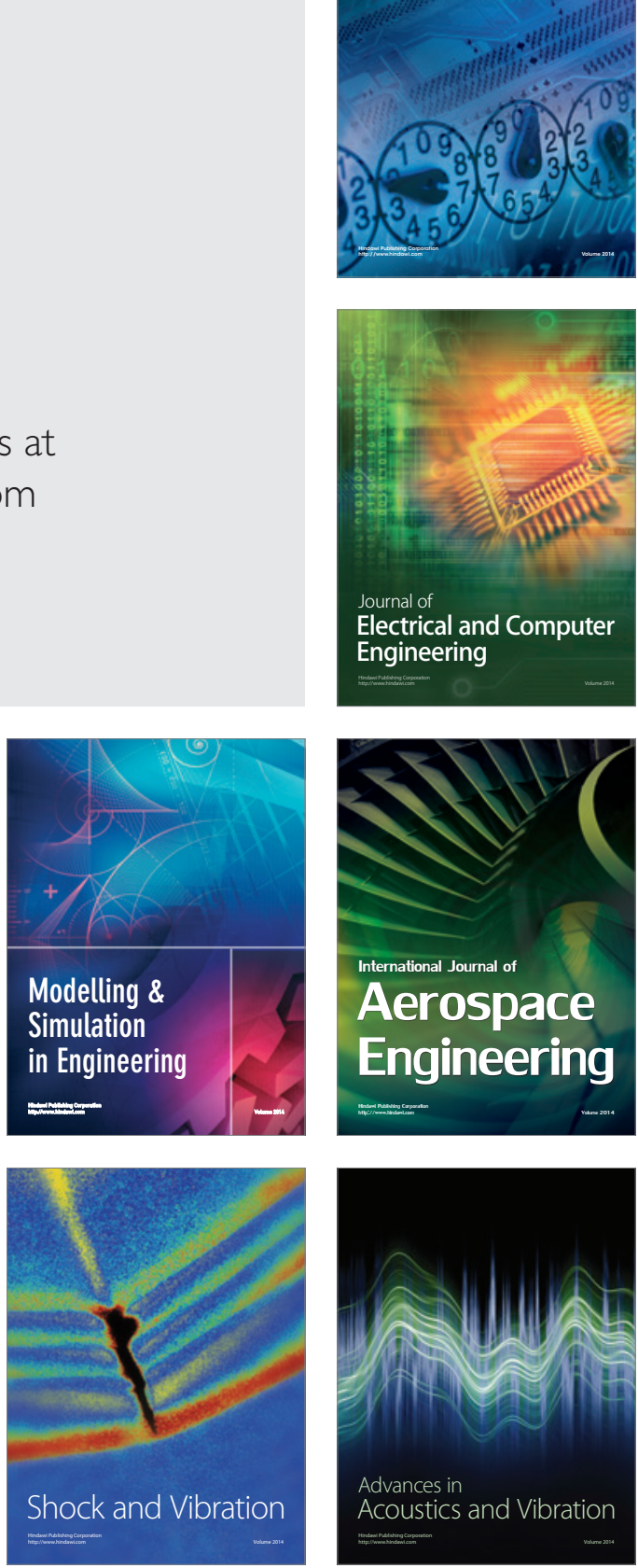